\title{
QoS-AODV6E: An Energy-Balancing QoS Routing Scheme for WSNs
}

\author{
Wolf-Bastian Pöttner, Oliver Wellnitz and Lars Wolf
}

\begin{abstract}
Wireless Sensor Networks (WSN) now advance into areas where new scenarios and applications require WSNs to meet certain Quality of Service $(\mathrm{Q} O S)$ requirements. To tackle these challenges, a routing approach has to consider limited energy supply of sensor nodes, dynamic network conditions as well as node mobility. This paper presents QoS-AODV6E, a routing scheme based on the Ad-hoc On-Demand Distance Vector (AODV) routing protocol, which encompasses QoS and Energy-Awareness for mobile WSNs. Based on the reactive AODV routing protocol, QoS-AODV6E allows applications to specify QoS parameters and finds suitable routes that support their requirements. In order to prolong the lifetime of the network, QoS-AODV6E balances the energy consumption between different paths through the network based on the weakest node. We evaluate our implementation of QoS-AODV6E in Contiki using simulation and measurements.
\end{abstract}

Index Terms-AODV, Routing, Energy, QoS, Measurements

\section{INTRODUCTION}

The development of small, low-power, resource-constrained, and radio-enabled devices has created a new and different area of applications. Combined with sensing and acting capabilities, these devices allow obtaining and distributing information in physical areas where no fixed infrastructure exists or the installation of such is either not feasible or not worthwhile. This paper concentrates on routing for such WSNs to monitor and control industrial plants. In this application, the network is sparsly populated, energy is tightly limited and the network must provide a certain minimum service quality.

We have chosen the reactive Ad-hoc On-Demand Distance Vector [1] (AODV) routing protocol as basis for our work. It is well suited for the use in dynamic WSNs [2] and features low processing overhead as well as exclusively local computation. Enhancing the metrics and mechanisms of AODV can tackle the challenges encountered in these networks. Since the basic mechanisms of Dynamic MANET On-demand [3] (DYMO) are similar to those of AODV, our results likewise also apply to it.

The contributions of this paper are as follows:

- QoS-AODV6E, an AODV-based routing protocol that supports IPv6, dynamic topologies, QoS signalling and energy-awareness

- An energy-aware routing metric that is based on the estimated lifetime of intermediate nodes

- An implementation of the routing protocol for Contiki

- Simulations and experimental evaluation

W.-B. Pöttner, O. Wellnitz and L. Wolf are with the Institute of Operating Systems and Computer Networks, Technische Universität Braunschweig, Germany. Email: [poettner|wellnitz|wolf] @ibr.cs.tu-bs.de

This work has been partially supported by the European Commission under the contract FP7-ICT-224282 (GINSENG).
The remainder of this paper is structured as follows: Section II discusses related research efforts. Section III describes the idea of QoS-AODV6E and the integration in AODV. In section IV, the implementation of QoS-AODV6E for Contiki is evaluated. Finally, the paper is concluded in section V.

\section{RELATED WORK}

For related work we focus on publications for WSNs or Mobile Ad-hoc Networks with routing mechanisms to tackle the problems of inhomogeneous energy consumption or network service quality.

MDR [4] uses the battery drain rate to estimate the residual lifetime. It then selects routes with the highest maximum lifetime value. Min-Max Battery Cost Routing (MMBCR) [5] selects a routing path in which the minimum residual energy is the highest. Conditional MMBCR switches between MMBCR and Minimum Battery Cost Routing (MBCR) to tackle the energy distribution problem in networks with diverse transmission powers and differing battery capacities. Maximum Residual Packet Capacity [6] (MRPC) builds on top of MMBCR and selects a routing path over which the highest number of packets can be transferred. $\max -\min z P_{\min }$ [7] uses a tradeoff between the lowest total network power consumption and the highest minimum residual power of the network. Dijkstra's algorithm is used to calculate the routes.

The main difference to our approach is that none of the presented papers uses the minimum residual lifetime of a routing path as a metric. In addition, none of theses proposals supports QoS.

\section{QOS-AODV6E}

We assume in this paper that all links are bidirectional and that nodes know their average local processing delay, the average medium access delay, the packet delivery rate per neighbour and the available bandwidth on the radio interface. We also assume a MAC protocol that switches off the radio whenever it is not needed.

\section{A. Energy-Aware Routing}

The idea of QoS-AODV6E is to prolong the lifetime of all nodes in the network by achieving an evenly distributed energy consumption between available nodes. To have a sufficiently accurate estimation of the node's lifetime under current load conditions, the EU-funded GINSENG [8] project develops novel mechanisms to accurately estimate the residual energy and the current consumption.

QoS-AODV6E first measures the minimum residual lifetime for each available routing path, whereas only paths with the 
least hop-count are considered. If more than one such path is available, energy-aware routing chooses the path in which the minimum residual lifetime is the highest. However, when the nodes start to transmit packets on that path, this causes additional energy consumption and therefore shortens the residual lifetime of involved nodes. Therefore it is important to constantly adapt to the changing energy situation. In order to do that, our energy-aware AODV uses two heuristic mechanisms: Firstly, the source node of a route counts the amount of incoming and outgoing data for that route. If the counter exceeds a threshold, the route will likely have a significantly changed energy situation and should be re-evaluated. Secondly, the involved intermediate nodes monitor their own residual lifetime. If the lifetime drops below a threshold compared to the lifetime at submission of the flow, the intermediate nodes notify the source to trigger a re-evaluation. In order to reevaluate the energy situation for a known route, the source node executes a completely new route discovery towards the destination node. Since the routing table entries are not touched, the data transmission can continue as usual during the rediscovery process.

In the route discovery cycle, the source node attaches a special extension to the outgoing route request (RREQ) message which encloses a field for the minimum residual lifetime along the routing path. Intermediate nodes update the field with the minimum of their own residual lifetime and the value found in the field. The destination node answers the first incoming RREQ message with a route reply (RREP) message. In contrast to the usual AODV behaviour, in energy-aware AODV the destination does not blindly discard subsequent RREQ messages for the same route discovery cycle. These new messages are compared with the already known route. If the message indicates a path with a higher minimum residual lifetime value for the same or a lower hop-count, the destination node issues an additional RREP message for the new path. Since the new RREP has a higher sequence number, this new route will overwrite the existing route.

An exception to these rules are nodes which are not energyconstrained since they are powered by power adaptors. The residual lifetime of such nodes can be modelled as infinitive; they will therefore never be the node with the least residual lifetime in a routing path. Energy-aware AODV uses routes only consisting of non-constrained nodes even with a higher hop-count, if the QoS requirements are fulfilled.

\section{B. Quality of Service Routing}

To assist the network in providing a certain service quality, our routing protocol can select appropriate routes. The approach taken in this paper is an in-band QoS signalling and admission control mechanism, thereby reducing the overhead and saving time in the route discovery cycle.

The solution presented in this paper is based on [9]-[11] and adapted it to the specialities of WSNs. The general idea is to enhance the existing route request (RREQ) messages with additional extensions, one for each QoS metric. The source node specifies the minimum requirements in this extension and intermediate nodes compare the requirement with their own abilities. If an intermediate node can fulfil the QoS request, it forwards the RREQ message as usual. If the intermediate node cannot fulfil the QoS request, it simply discards the RREQ message. This ensures that the destination node only receives RREQ messages for paths in which all intermediate nodes and links fulfil the QoS requirements. This model of a distributed admission control avoids the need for frequent link state updates and still delivers accurate QoS information. In typical application scenarios for WSNs, there are three distinct metrics to specify the service quality.

The source node can specify the maximum end-to-end delay in the RREQ message. Intermediate nodes subtract their average local processing delay and their average medium access delay from that value. If the residual value is zero or below, the intermediate nodes drop the route request message, otherwise they update and forward the message as usual.

The source node can specify the minimum bandwidth required for that specific flow in the RREQ message. Intermediate nodes need to check if there is enough capacity for that new flow on the interface. If the intermediate node does not have enough bandwidth available, the route request message is discarded. Otherwise it is forwarded as usual.

The reliability of a path through the network can be characterised as the ratio of successfully delivered packets compared to the total number of sent packets. Since the end-to-end reliability depends on the reliability of each involved link, it can be seen as the combination of the link reliabilities [12], if those are independent. The source node can specify the minimum required packet delivery rate (PDR) in an extension to the RREQ message. The extension has a second field to hold the current PDR, initialised to $100 \%$. Intermediate nodes multiply their own PDR with the field value and update the extension with this new, combined value. If the combined value is less than the minimum specified value, the intermediate node drops the route request message.

In addition, an extension allows to specify an identifier for each individual flow.

When QoS-based and energy-aware AODV are used together, side effects occur. If the rediscovery leads to a changed route between source and destination, the QoS parameters of the route will inevitably also change. The QoS characteristics of a route should therefore be seen as equal or better than the specified minimum.

\section{Implementation}

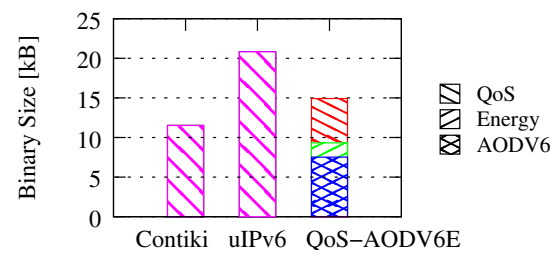

Figure 1. Flash consumption of different components of QoS-AODV6E compared to Contiki and uIPv6.

The implementation of QoS-AODV6E is crafted for the Contiki [13] operating system and relies on the uIPv6 stack. 


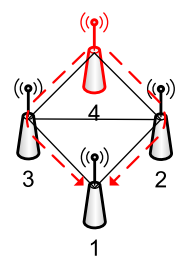

(a) Measurement setup for energy-aware AODV

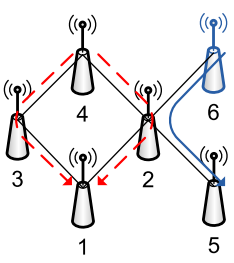

(b) Simulation setup for QoS AODV
Figure 2. Network setups to evaluate QoS-AODV6E.

QoS-AODV6E integrates with the existing routing interface in Contiki and does not need explicit application support for basic functionality. For QoS-based and energy-aware routing, QoS-AODV6E offers additional interfaces to the developer to configure the necessary parameters. The design of QoSAODV6E is modular to enable users to configure it to the exact needs of the specific application and save valuable space in flash. The flash consumption of QoS-AODV6E in different configurations can be seen in Figure 1.

A problem of Contiki is that it does not support queuing of packets and that the radio drivers do not know the average medium access delays, the available radio bandwidth and the average packet delivery rate per neighbour. Because of these limitations of Contiki, this paper relies on simple mechanisms to estimate these values.

\section{Evaluation}

Since AODV has been published in 2003 and evaluated numerous times since then, we concentrate on evaluating the additional features presented in this paper and compare them with the performance of standard AODV.

To have comparable amounts of residual energy for all nodes, this paper uses a simulated energy resource. Each node has the same amount of residual energy on start-up and consumes energy per time interval and per incoming and outgoing byte. When this simulated value reaches 0 , the node stops responding and cannot forward packets anymore. This behaviour resembles the energy signature of MAC protocols that switch off the radio between transmissions. One example of such a MAC protocol is X-MAC [14].

The first step of our evaluation is to define metrics to evaluate the performance of QoS-AODV6E. The longer a node lives, the longer it can fulfil its duties. Since the network may suffer from a split after a node failure, each node shall stay operational as long as possible. Consequently, the lifetime of the network is defined as the minimum of the lifetimes of all nodes.

Another important metric in the focus of this paper is the network quality. This evaluation concentrates on packet loss for a specific data flow, since intermediate nodes that cannot handle the bandwidth of all flows will drop packets.

\section{A. Measurements With Energy-Aware AODV}

The network was setup as seen in Figure 2(a) and consists of AVR Raven nodes. In this measurement, node 4 sends 5 packets per second with a UDP payload size of 63 bytes to

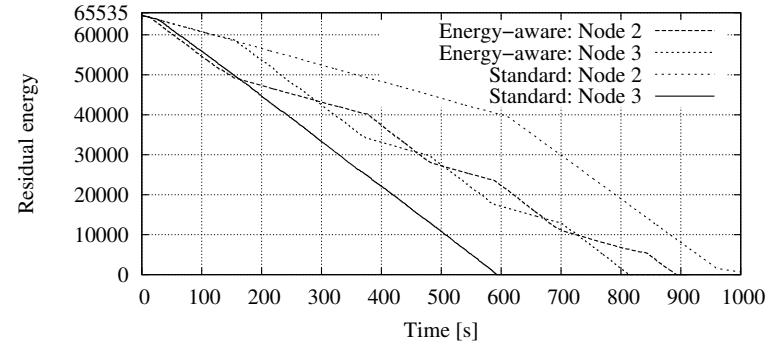

Figure 3. Residual Lifetime of nodes with standard and energy-aware AODV.

\begin{tabular}{|l||r|r|r||c|}
\hline \multicolumn{1}{|l||}{ Measurement } & 1 & 2 & 3 & Avg. \\
\hline \hline \multicolumn{1}{|c||}{} & \multicolumn{4}{c|}{ Standard AODV } \\
\hline Lifetime Intermediate 2 & $(*) 965 \mathrm{~s}$ & $932 \mathrm{~s}$ & $(*) 974 \mathrm{~s}$ & $957 \mathrm{~s}$ \\
\hline Lifetime Intermediate 3 & $577 \mathrm{~s}$ & $543 \mathrm{~s}$ & $592 \mathrm{~s}$ & $571 \mathrm{~s}$ \\
\hline Network Lifetime & $\mathbf{5 7 7} \mathrm{s}$ & $\mathbf{5 4 3} \mathrm{s}$ & $\mathbf{5 9 2} \mathrm{s}$ & $\mathbf{5 7 1 ~ s}$ \\
\hline \hline \multicolumn{1}{|c||}{} & \multicolumn{5}{c|}{ Energy aware AODV } \\
\hline Lifetime Intermediate 2 & $782 \mathrm{~s}$ & $892 \mathrm{~s}$ & $894 \mathrm{~s}$ & $856 \mathrm{~s}$ \\
\hline Lifetime Intermediate 3 & $779 \mathrm{~s}$ & $817 \mathrm{~s}$ & $852 \mathrm{~s}$ & $816 \mathrm{~s}$ \\
\hline Network Lifetime & $\mathbf{7 7 9} \mathrm{s}$ & $\mathbf{8 1 7} \mathrm{s}$ & $\mathbf{8 5 2} \mathrm{s}$ & $\mathbf{8 1 6} \mathrm{s}$ \\
\hline
\end{tabular}

Table I

LIFETIMES OF INTERMEDIATE NODES IN ALL MEASUREMENT RUNS.

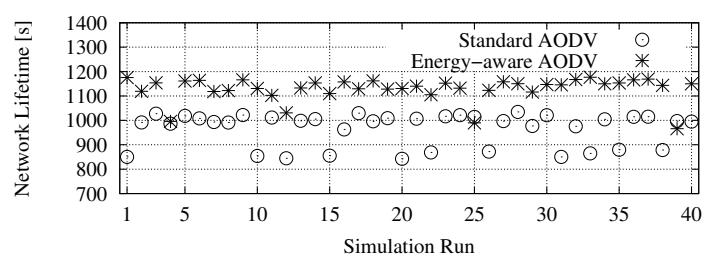

Figure 4. Network lifetimes for standard and energy-aware AODV.

node 1 . This results in the possible maximum of 128 bytes radio packets without fragmentation. The rediscovery interval of energy-aware routing was configured to 500 IP packets and each measurement was conducted at least 3 times to verify the results.

Figure 3 visualises the effect of energy-aware routing. It can be seen that for standard AODV, intermediate node 2 suddenly consumes less energy. This phenomenon is caused by the sender node whose batteries were depleted. Under the previous load conditions, intermediate node 2 would have failed at $974 \mathrm{~s}$. In this exemplary measurement, the lifetime of the network is $592 \mathrm{~s}$ for standard and $817 \mathrm{~s}$ for energyaware AODV. This means that the network lifetime can be improved by $38 \%$ with energy-aware AODV. The maximum intermediate node lifetime is $974 \mathrm{~s}$ for standard and $892 \mathrm{~s}$ for energy-aware AODV. This reduced maximum lifetime is caused by overhead for additional route discovery cycles and resembles a decrease of $8 \%$. Table I gives an overview over three measurements for standard and energy-aware AODV. The lifetimes marked with an asterisk are interpolated values since the sender node failed earlier than the second intermediate node due to depleted batteries. The table shows that the average network lifetime can be improved by $43 \%$ by using energy-aware AODV. 


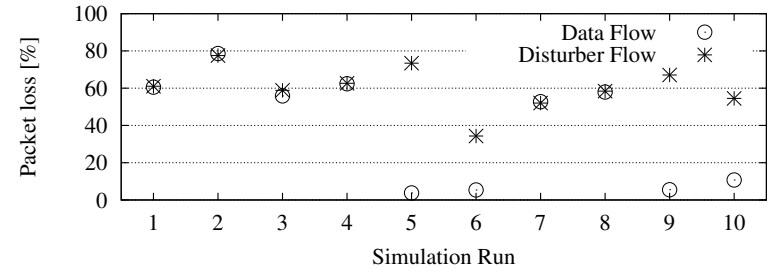

(a) Standard AODV

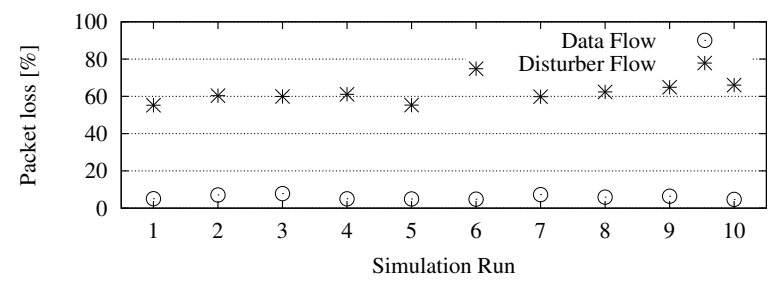

(b) QoS-based AODV

Figure 5. Overall packet loss for standard and QoS-based AODV.

\section{B. Simulations With Energy-Aware AODV}

Figure 4 shows the results of simulations comparing standard and energy-aware AODV for a network of five nodes. In this case, two nodes send a total of 3 packets with 63 bytes UDP payload per second to one receiver. It can be seen, that energy-aware AODV can improve the lifetime of the network by $17 \%$ in average.

\section{Simulations With Quality of Service Based AODV}

To simulate the evaluation networks we have used Cooja with the Unit Disc Graph radio model and the JNI-based native compilation method. The network setup can be seen in Figure 2(b). In this simulation, node 4 sends 4 packets per second containing 63 bytes of UDP payload to node 1 . In addition to that there is a disturbing background flow of the same rate and volume from node 6 to node 5 . In the QoS-enabled simulation, the former flow requests a minimum bandwidth from QoS-AODV6E.

Figure 5 shows the overall packet loss of the two flows for standard and QoS-based AODV. For standard AODV, Subfigure (a) shows two complementary behaviours. In 4 simulations, the data flow has a packet loss below $15 \%$ since AODV chose to route packets from node 4 over node 3 , thereby avoiding the bottleneck at node 2 . However, in the remaining simulations the data flow has a packet loss above $50 \%$. In all simulations, the disturber flow has an unacceptable high packet loss. In contrast, the packet loss of the data flow for QoS-based AODV in Subfigure (b) is steady between 4 and $8 \%$. This low value is caused by the fact that QoSbased AODV always chose node 3 as intermediate hop for packets between nodes 4 and 1 . However, the packet loss of the disturber flow is still at the high level seen for standard AODV since no alternative path exists. The outcome of this simulation is that QoS-based routing can reduce the overall average packet loss of the data flow by $85 \%$ by selecting a suitable path. However, the packet loss can only be influenced if suitable paths exist.

\section{CONCLUSION}

Upcoming applications require WSNs to meet applicationspecific performance targets, to support node mobility and to deal efficiently with the scarce availability of energy. This paper proposes a new routing approach based on AODV, that balances energy consumption between alternating paths by incorporating the minimum residual lifetime of the paths. Thereby, the lifetime of the whole network can be prolonged. To meet certain performance targets, the selection of paths that fulfil these requirements can grant service quality to applications that need it. By supporting local route repair, nodes can be mobile and still communicate with the network. The performance evaluation has shown that the proposed mechanisms work and that the results are encouraging. Results that were ommited here due to limitations in space can be found in [15]. Further research work has to evaluate QoSAODV6E in more complex network settings.

\section{REFERENCES}

[1] C. Perkins, E. Belding-Royer, and S. Das, "Ad hoc On-Demand Distance Vector (AODV) Routing,” Internet Draft, United States, Jul. 2003.

[2] S. H. Manjula, C. N. Abhilash, K. Shaila, K. R. Venugopal, and L. M. Patnaik, "Performance of AODV Routing Protocol using Group and Entity Mobility Models in Wireless Sensor Networks," in Proceedings of the International MultiConference of Engineers and Computer Scientists, vol. 2, Hong Kong, Mar. 2008.

[3] I. Chakeres and C. Perkins, "Dynamic MANET On-demand (DYMO) Routing," Internet Draft, Mar. 2010.

[4] D. Kim, J. J. Garcia-Luna-Aceves, K. Obraczka, J.-C. Cano, and P. Manzoni, "CMDR: Conditional Minimum Drain Rate Protocol for Route Selection in Mobile Ad-Hoc Networks," in Proceedings of the International Conference on Information Networking, Cheju Island, Korea, Feb. 2003, pp. 702-712.

[5] C.-K. Toh, "Maximum Battery Life Routing to Support Ubiquitous Mobile Computing in Wireless Ad Hoc Networks," IEEE Communications Magazine, vol. 39, no. 6, pp. 138-147, Jun. 2001.

[6] A. Misra and S. Banerjee, "MRPC: maximizing network lifetime for reliable routing in wireless environments," in Proceedings of the Wireless Communications and Networking Conference (WCNC), vol. 2, Orlando, Florida, USA, Mar. 2002, pp. 800-806.

[7] Q. Li, J. Aslam, and D. Rus, "Hierarchical power-aware routing in sensor networks," in In Proceedings of the DIMACS Workshop on Pervasive Networking, Piscataway, New Jersey, USA, May 2001.

[8] C. Sreenan, J. S. Silva, L. Wolf, R. Eiras, T. Voigt, U. Roedig, V. Vassiliou, and G. Hackenbroich, "Performance Control in Wireless Sensor Networks: The Ginseng Project - [Global Communications News Letter]," Communications Magazine, vol. 47, no. 8, p. 1, Aug. 2009.

[9] L. Chen and W. Heinzelman, "QoS-Aware Routing Based on Bandwidth Estimation for Mobile Ad Hoc Networks," IEEE Journal on Selected Areas in Communications, vol. 23, no. 3, pp. 561-572, March 2005.

[10] Q. Xue and A. Ganz, "Ad hoc QoS on-demand routing (AQOR) in mobile ad hoc networks," Journal of Parallel and Distributed Computing, vol. 63, no. 2, pp. 154-165, Feb. 2003.

[11] C. E. Perkins, E. M. Royer, and S. R. Das, "Quality of Service for Ad hoc On-Demand Distance Vector Routing," Internet Draft, Nov. 2001.

[12] K. Shin, M. Y. Chung, J. Won, and H. Choo, "Routing Based on Ad Hoc Link Reliability," in Proceedings of ISPA Workshops, Sorrento, Italy, Dec. 2006, pp. 341-350.

[13] A. Dunkels, B. Grönvall, and T. Voigt, "Contiki - a Lightweight and Flexible Operating System for Tiny Networked Sensors," in Proceedings of the First IEEE Workshop on Embedded Networked Sensors (EmnetsI), Tampa, Florida, USA, Nov. 2004.

[14] M. Buettner, G. V. Yee, E. Anderson, and R. Han, "X-MAC: a short preamble MAC protocol for duty-cycled wireless sensor networks," in Proceedings of the 4th international conference on Embedded networked sensor systems, New York, NY, USA, 2006, pp. 307-320.

[15] W.-B. Pöttner, "A QoS-based Routing Protocol for Dynamic Wireless Sensor Networks," Master's thesis, TU Braunschweig, Jun. 2009. 